\title{
Screening without screening: Baryon energy at high baryon density
}

\author{
Jeff Greensite and Evan Owen \\ Physics and Astronomy Department, San Francisco State University, \\ San Francisco, California 94132, USA
}

(Received 12 May 2019; published 5 August 2019)

\begin{abstract}
We compute the Coulomb interaction energy of dense sets of static quarks in a compact volume (much smaller than the lattice volume) containing one quark per lattice site. The quark color charges are combined into either a set of three-quark nucleon states, or into a nonfactorizable "one big hadron" state. In both cases we find that the energy per quark is roughly constant as the volume of quarks increases. A surprise is that if we construct the nucleon states from sets of three quarks chosen at random in the volume, then the energy per quark remains roughly constant, even as the average distance between quarks in a nucleon state grows as the volume increases. This energy dependence of a nucleon in a dense medium is at odds with the behavior of an isolated nucleon as quark separation increases, and for static quarks it is not easily explicable in terms of some version of Debye screening.
\end{abstract}

DOI: 10.1103/PhysRevD.100.034503

\section{INTRODUCTION}

The study of QCD at high baryon density is constrained by the (as yet) unsolved sign problem. There are, however, some situations related to QCD at high baryon density where the sign problem is less acute. In this article we will study the Coulomb interaction energy of a dense system of static quark charges in a fixed volume, and for this problem we will see that standard Monte Carlo methods will suffice. The thermodynamics of a system of heavy dense quarks at finite chemical potential has already been treated by other (e.g., Langevin) methods [1,2], but our focus here is a little different and goes a little beyond the phase diagram. Rather than introducing a chemical potential and having a finite baryon density throughout the lattice volume, we will place $2^{p} \times 3$ quark charges in a subvolume of the lattice, with a density of one quark per site. This requires that the color charges are contracted into an overall color singlet combination, and we will consider two types of contractions:

(1) A "multinucleon" (MN) state. This consists of division of the quarks into $2^{p}$ sets of three quarks (not necessarily nearest neighbors), and contraction of the quark charges in each set into a color singlet. This leaves $2^{p}$ "nucleon" states.

(2) A "diquark pyramid" (DQP) state. Here we first construct $2^{p-1} \times 3$ diquark states in the $\overline{\mathbf{3}}$ representation, form from these $2^{p-2} \times 3$ sets of states in the 3

Published by the American Physical Society under the terms of the Creative Commons Attribution 4.0 International license. Further distribution of this work must maintain attribution to the author(s) and the published article's title, journal citation, and DOI. Funded by SCOAP ${ }^{3}$. representation, and so on until we arrive at three states in either the $\mathbf{3}$ (even $p$ ) or $\overline{\mathbf{3}}$ (odd $p$ ) representations, which are finally contracted into a singlet.

The point to notice is that unlike the MN state, a DQP state cannot be factorized into two or more subsets of color singlets. It is, in a sense, one big hadron, where every quark interacts in some way with every other quark.

Our focus in this article is on energetics. We would like to know how the color Coulomb interaction energy per quark depends on volume, on how the colors are contracted, and on the average distance (in the MN case) between quarks in a nucleon. One important point to note from the beginning is that the usual mechanisms of charge screening in a plasma are not available here, because the charges are static, and the positions of the quarks are fixed.

A theory with only massive static quarks (and hence with negligible quark loop effects) is not immune from the sign problem, which is tied to finite baryon density. And one indeed has a sign problem in the standard formulation with a chemical potential, in the well-studied heavy-dense limit (see, e.g., $[1,2])$ where the effect of quark loops is absent. In the type of canonical approach we follow here, the sign problem must manifest itself through strong oscillations in the sign of the observable. But we will find, for the observables from which we extract the Coulomb energy, that these sign oscillations are manageable. One could of course repeat our calculations using gauge field configurations generated with light dynamical quarks, but with a finite baryon density due to heavy static quarks only. We leave this for future investigation.

\section{COLOR CONTRACTIONS}

In this section we use an upper-lower color index convention to distinguish between indices transforming 
in the $\overline{\mathbf{3}}$ and $\mathbf{3}$ representations, respectively, with indices raised and lowered by complex conjugation. Thus a gauge transformation $g(x)$ transforms quark fields as

$$
\begin{aligned}
\psi_{a}(x) \rightarrow \psi_{a}^{\prime}(x) & =g_{a}{ }^{b}(x) \psi_{b}(x) \\
\bar{\psi}^{a}(x) \rightarrow \bar{\psi}^{\prime a}(x) & =g^{a}{ }_{b}(x) \bar{\psi}^{b}(x) \\
g_{a}{ }^{b}(x) g^{c}{ }_{b}(x) & =g^{b}{ }_{a}(x) g_{b}{ }^{c}(x)=\delta_{a}^{c} .
\end{aligned}
$$

In a theory with local SU(3) gauge invariance, a set of quark operators $\psi_{a}$ in the equal-times combination ${ }^{1}$

$$
\epsilon^{a b c} \psi_{a}\left(\boldsymbol{x}_{1}\right) \psi_{b}\left(\boldsymbol{x}_{2}\right) \psi_{c}\left(\boldsymbol{x}_{3}\right)
$$

is invariant under global, but not local SU(3) gauge transformations. To form a gauge-invariant combination which, when applied to the vacuum, would create a physical state, we must in general replace $\epsilon_{a b c}$ by a multicovariant gluonic operator

$$
V^{a b c}\left(\boldsymbol{x}_{1}, \boldsymbol{x}_{2}, \boldsymbol{x}_{3} ; A\right) \psi_{a}\left(\boldsymbol{x}_{1}\right) \psi_{b}\left(\boldsymbol{x}_{2}\right) \psi_{c}\left(\boldsymbol{x}_{3}\right),
$$

which is a functional of the gauge field $A$ on a time slice, and which transforms under a local gauge transformation $g$ as

$$
\begin{aligned}
V^{a b c} & \left(\boldsymbol{x}_{1}, \boldsymbol{x}_{2}, \boldsymbol{x}_{3} ; A\right) \\
& \rightarrow V^{\prime a b c}\left(\boldsymbol{x}_{1}, \boldsymbol{x}_{2}, \boldsymbol{x}_{3} ; A\right) \\
\quad & =g^{a}{ }_{a^{\prime}}\left(\boldsymbol{x}_{1}\right) g^{b}{ }_{b^{\prime}}\left(\boldsymbol{x}_{2}\right) g_{c^{\prime}}^{c}\left(\boldsymbol{x}_{3}\right) V^{a^{\prime} b^{\prime} c^{\prime}}\left(\boldsymbol{x}_{1}, \boldsymbol{x}_{2}, \boldsymbol{x}_{3} ; A\right) .
\end{aligned}
$$

This obviously generalizes to any number of quarks (provided that number is divisible by 3 ); i.e., the creation operator for a system of $N$ quarks will have the form

$$
\begin{aligned}
& V^{a_{1} a_{2} a_{3} \ldots a_{N}}\left(\boldsymbol{x}_{1}, \boldsymbol{x}_{2}, \boldsymbol{x}_{3}, \ldots, \boldsymbol{x}_{N} ; A\right) \\
& \quad \times \psi_{a_{1}}\left(\boldsymbol{x}_{1}\right) \psi_{a_{2}}\left(\boldsymbol{x}_{2}\right) \psi_{a_{3}}\left(\boldsymbol{x}_{3}\right) \ldots \psi_{a_{N}}\left(\boldsymbol{x}_{N}\right) .
\end{aligned}
$$

What is meant by a "multinucleon" state is that the operator which creates the state can be factorized into products of gauge invariant operators, each composed of three quarks. So the operator which creates a MN state of six quarks factorizes into a product of two nucleon operators, e.g.,

$$
\begin{aligned}
\Psi^{\mathrm{MN}}= & V^{a_{1} a_{2} a_{3} a_{4} a_{5} a_{6}}\left(\boldsymbol{x}_{1}, \boldsymbol{x}_{2}, \boldsymbol{x}_{3}, \boldsymbol{x}_{4}, \boldsymbol{x}_{5}, \boldsymbol{x}_{6}\right) \psi_{a_{1}}\left(\boldsymbol{x}_{1}\right) \\
& \times \psi_{a_{2}}\left(\boldsymbol{x}_{2}\right) \psi_{a_{3}}\left(\boldsymbol{x}_{3}\right) \psi_{a_{4}}\left(\boldsymbol{x}_{4}\right) \psi_{a_{5}}\left(\boldsymbol{x}_{5}\right) \psi_{a_{6}}\left(\boldsymbol{x}_{6}\right) \Psi_{0} \\
= & \left\{V^{a_{1} a_{2} a_{3}}\left(\boldsymbol{x}_{1}, \boldsymbol{x}_{2}, \boldsymbol{x}_{3}\right) \psi_{a_{1}}\left(\boldsymbol{x}_{1}\right) \psi_{a_{2}}\left(\boldsymbol{x}_{2}\right) \psi_{a_{3}}\left(\boldsymbol{x}_{3}\right)\right\} \\
& \times\left\{V^{a_{4} a_{5} a_{6}}\left(\boldsymbol{x}_{4}, \boldsymbol{x}_{5}, \boldsymbol{x}_{6}\right) \psi_{a_{4}}\left(\boldsymbol{x}_{4}\right) \psi_{a_{5}}\left(\boldsymbol{x}_{5}\right) \psi_{a_{6}}\left(\boldsymbol{x}_{6}\right)\right\} \Psi_{0},
\end{aligned}
$$

where $\Psi_{0}$ is the vacuum state. A DQP operator cannot be factorized in this manner.

\footnotetext{
${ }^{1}$ We ignore spin states and Dirac indices; for the Coulomb energy only color combinations are important.
}

We are interested in comparing the energies of $\mathrm{MN}$ and DQP states. Of course it is difficult to do this in full generality, not least because the $V$ operators which minimize the energies of such states are unknown. However, if we are satisfied with just computing numerically the Coulomb interaction energy of such states, then it is possible to make such comparisons. By "Coulomb energy" we mean the energy, above the vacuum energy, of a state obtained from a set of quark creation operators in Coulomb gauge acting on the vacuum. In particular, let $A_{k}(\boldsymbol{x})$ be a gauge field at some fixed time $t$, and let $g(x ; A)$ be the gauge transformation which takes a quark operator at point $x$ into Coulomb gauge. Then we may construct, e.g., a gauge-invariant one-nucleon state as

$$
\begin{aligned}
\Psi= & \epsilon^{a_{1} a_{2} a_{3}} g_{a_{1}}^{b_{1}}\left(\boldsymbol{x}_{1} ; A\right) g_{a_{2}}{ }^{b_{2}}\left(\boldsymbol{x}_{2} ; A\right) g_{a_{3}}{ }^{b_{3}}\left(\boldsymbol{x}_{3} ; A\right) \\
& \times \psi_{b_{1}}\left(\boldsymbol{x}_{1}\right) \psi_{b_{2}}\left(\boldsymbol{x}_{2}\right) \psi_{b_{3}}\left(\boldsymbol{x}_{3}\right) \Psi_{0} .
\end{aligned}
$$

If $A$ is already in Coulomb gauge, then $g(\boldsymbol{x} ; A)$ is the identity operator, and therefore in Coulomb gauge we have simply

$$
\Psi=\epsilon^{a_{1} a_{2} a_{3}} \psi_{a_{1}}\left(\boldsymbol{x}_{1}\right) \psi_{a_{2}}\left(\boldsymbol{x}_{2}\right) \psi_{a_{3}}\left(\boldsymbol{x}_{3}\right) \Psi_{0} .
$$

We note that Coulomb gauge does not fix the gauge uniquely (even if we ignore the Gribov copy issue), because the gauge condition is preserved by gauge transformations $g(\boldsymbol{x}, t)=g(t)$ which are constant on a time slice. The contraction of indices with the Levi-Civita tensor is required in order that $\Psi$ be invariant under this global remnant of the gauge symmetry. The interaction energy of a state of this kind can only be due to the nonlocal Coulomb term in the Hamiltonian, since there is no other expression in the Coulomb gauge Hamiltonian which could give rise to an interaction energy between spatially separated quarks. So we will refer to the energy of state (8) above the vacuum energy as the "Coulomb energy" of a set of three static quarks.

More generally, let $S^{\dagger}$ be the creation operator for a set of static quarks in Coulomb gauge, invariant under the remnant symmetry, and denote

$$
\left|\Psi_{S}\right\rangle=S^{\dagger}\left|\Psi_{0}\right\rangle .
$$

We define $\mathcal{E}_{S}(t)$, on a Euclidean lattice with discretized time, from the vacuum expectation values

$$
\begin{aligned}
e^{-\mathcal{E}_{S}(t)} & \equiv \frac{\left\langle S(t+1) S^{\dagger}(0)\right\rangle}{\left\langle S(t) S^{\dagger}(0)\right\rangle} \\
& =\frac{\left\langle\Psi_{S}\left|e^{-\left(H-E_{0}\right)(t+1)}\right| \Psi_{S}\right\rangle}{\left\langle\Psi_{S}\left|e^{-\left(H-E_{0}\right) t}\right| \Psi_{S}\right\rangle},
\end{aligned}
$$

where by $e^{-H n}$ we mean the $n$th power of the transfer matrix. Note that 


$$
\mathcal{E}_{S}^{\min }=\lim _{t \rightarrow \infty} \mathcal{E}_{S}(t)
$$

is the minimum possible energy, above the vacuum energy $E_{0}$, of a state containing the same set of static quarks as $\Psi_{S}$, in the same spatial positions with the same color contractions. At the other end of the timescale, in the $t=0$ limit,

$$
\mathcal{E} \equiv \mathcal{E}_{S}(0)
$$

may be regarded as a definition of the Coulomb energy of state $\Psi_{S}$ on a discrete time lattice, and it is energies of this kind that we report below. We of course compute $\mathcal{E}$ on a periodic lattice at each time slice $t$ and average over time slices, so that the quantities to be computed by lattice Monte Carlo are $\left\langle S(t+1) S^{\dagger}(t)\right\rangle$.

The integration over static quark fields in the $S(t+$ 1) $S^{\dagger}(t)$ operator leaves us with a set of timelike link variables on a time slice, with one link variable for each static quark position running between times $t$ and $t+1$, and with indices contracted to form a singlet under the remnant global symmetry. As an example, for the one nucleon state in (8), we have for the Coulomb energy

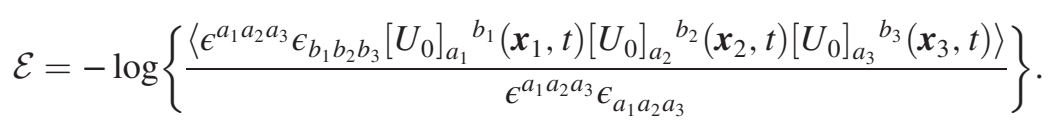

The generalization to larger sets of quarks is straightforward. In Coulomb gauge the $V$ operators are simply tensors, independent of position and gauge field, which contract quark indices into global color singlets, and the Coulomb energy is computed on the lattice from the correlators

$$
\mathcal{E}=-\log \left\{\frac{\left\langle V^{a_{1} a_{2} \ldots a_{N}} V_{b_{1} b_{2} \ldots b_{N}}\left[U_{0}\right]_{a_{1}}^{b_{1}}\left(\boldsymbol{x}_{1}, t\right)\left[U_{0}\right]_{a_{2}}{ }^{b_{2}}\left(\boldsymbol{x}_{2}, t\right) \ldots\left[U_{0}\right]_{a_{N}}^{b_{N}}\left(\boldsymbol{x}_{N}, t\right)\right\rangle}{V^{a_{1} a_{2} \ldots a_{N}} V_{a_{1} a_{2} \ldots a_{N}}}\right\}
$$

evaluated in Coulomb gauge. In the past this method has been used to compute the Coulomb energy of a single quark-antiquark pair as a function of quark separation $[3,4]$. Our intention here is to apply the same technique to a dense system of quarks.

\section{A. Gauge invariance and Coulomb energy}

Although the expression for Coulomb energy boils down to computing the expectation value of a certain product of timelike link variables in Coulomb gauge, it is important to recognize that this is the computation of a gauge invariant quantity, namely the energy of a certain physical state, which happens to have an especially simple form when evaluated in Coulomb gauge. Because of the apparent gauge dependence of (14), this point may be worth emphasizing. For simplicity, consider a gauge invariant quark-antiquark state of the form

$$
\Psi_{\bar{\psi} \psi}=\bar{\psi}^{a}(\boldsymbol{x}) V_{a}^{b}(\boldsymbol{x}, \boldsymbol{y} ; U) \psi_{b}(\boldsymbol{y}) \Psi_{0},
$$

where $V$ transforms like a Wilson line running between sites $\boldsymbol{x}, \boldsymbol{y}$, at some fixed time $t$, and moreover $V$ depends only on spacelike link variables $U$ at the same fixed time $t$. $\Psi_{0}$ is the ground state. $\Psi_{\bar{\psi} \psi}$ satisfies Gauss's law, and it is a physical state, with an energy given by the lattice logarithmic derivative

$$
\mathcal{E}=-\log \left[\frac{\left\langle\operatorname{Tr}\left[U_{0}(\boldsymbol{x}, 0) V(\boldsymbol{x}, \boldsymbol{y}, t ; U) U^{\dagger}(\boldsymbol{y}, 0) V(\boldsymbol{y}, \boldsymbol{x}, 0 ; U)\right]\right\rangle}{\langle\operatorname{Tr}[V(\boldsymbol{x}, \boldsymbol{y}, 0 ; U) V(\boldsymbol{y}, \boldsymbol{x}, 0 ; U)]\rangle}\right],
$$

where $V(\boldsymbol{x}, \boldsymbol{y}, t ; U)$ is the $V$ operator evaluated from spacelike links $U$ at time $t$. There are, however, an infinite number of possible $V$ operators with the appropriate transformation properties. One possibility, for $\boldsymbol{x}, \boldsymbol{y}$ lying along, e.g., the $x$-axis, is to choose $V$ to be a Wilson line running between these points. The physical state $\Psi_{\bar{\psi} \psi}$ then represents a static quark and antiquark with a thin line of color electric flux running between them, and $\mathcal{E}$, which is the energy of this state, involves taking the logarithm of a familiar object, namely an $R \times T$ rectangular Wilson loop with $T=1$. The gauge invariance of such a quantity is obvious, but it must be recognized that this is only one possible physical state. There are other ways to satisfy Gauss's law, and one of them is to create, along with the static sources, a longitudinal color electric field which solves Gauss's law in the presence of those static sources. We are led to consider, instead of a Wilson line, the following operator:

$$
V_{a}^{b}(\boldsymbol{x}, \boldsymbol{y})=g^{c}{ }_{a}(\boldsymbol{x} ; U) \delta_{c}^{d} g_{d}^{b}(\boldsymbol{y} ; U),
$$

where $g_{a}{ }^{b}(\boldsymbol{x} ; U)$ is the gauge transformation which takes the link variables at fixed time $t$ into Coulomb gauge. It is easy to check that under an arbitrary gauge transformation $g^{\prime}$, 


$$
\begin{aligned}
g_{a}{ }^{b}(\boldsymbol{x} ; U) & \rightarrow g_{a}{ }^{c}(\boldsymbol{x} ; U) g^{\prime b}{ }_{c}(\boldsymbol{x}) \\
g^{a}{ }_{b}(\boldsymbol{x} ; U) & \rightarrow g^{\prime}{ }_{b}{ }^{c}(\boldsymbol{x}) g^{a}{ }_{c}(\boldsymbol{x} ; U),
\end{aligned}
$$

which establishes the gauge covariance of the $V$ operator in (17), and the gauge invariance of the corresponding energy. As it happens, this construction of the $V$ operator in the Abelian theory yields, for static opposite charges, the minimal energy state $(\bar{\psi} V \psi) \Psi_{0}$ of the theory. The $V$ operator in (17) takes on a very simple form in Coulomb gauge, where $g(\boldsymbol{x} ; U)=\mathbb{1}$, and therefore $V_{a}{ }^{b}(\boldsymbol{x}, \boldsymbol{y})=\delta_{a}^{b}$. Computing the energy of the corresponding physical state just requires computing the correlator of timelike link variables $\left\langle\operatorname{Tr}\left[U_{0}(\boldsymbol{x}) U_{0}^{\dagger}(\boldsymbol{y})\right]\right.$ on a time slice. Although the expression does not look gauge invariant when evaluated in Coulomb gauge (as opposed, e.g., to a rectangular Wilson loop), it must be recognized that in fact it is nothing more than the Coulomb gauge version of the gauge invariant expression (16) with the covariant $V$ operator (17). After fixing to Coulomb gauge, the Kronecker delta in (17) contracts indices into a singlet under the remnant global gauge transformations, which are constant on a time slice. In passing to more complicated physical states, such as those involving large numbers of static quarks, the simple Kronecker delta is replaced by other tensors, such as the Levi-Civita tensor, which have the same purpose in contracting indices to form a singlet under global transformations. As an example, we create a physical one-nucleon state with the gauge invariant operator shown in (3), and we specialize to a subclass of states for which

$$
\begin{aligned}
& V^{b_{1} b_{2} b_{3}}\left(\boldsymbol{x}_{1}, \boldsymbol{x}_{2}, \boldsymbol{x}_{3} ; U\right) \\
& \quad=\epsilon^{a_{1} a_{2} a_{3}} g_{a_{1}}{ }^{b_{1}}\left(\boldsymbol{x}_{1} ; U\right) g_{a_{2}}{ }^{b_{2}}\left(\boldsymbol{x}_{2} ; U\right) g_{a_{3}}{ }^{b_{3}}\left(\boldsymbol{x}_{3} ; U\right) .
\end{aligned}
$$

In Coulomb gauge, this expression simplifies to $V^{b_{1} b_{2} b_{3}}\left(\boldsymbol{x}_{1}\right.$, $\left.\boldsymbol{x}_{2}, \boldsymbol{x}_{3} ; U\right)=\epsilon^{b_{1} b_{2} b_{3}}$.

Thus the apparent gauge dependence of (14) should not be construed as some violation of gauge invariance. It is simply the Coulomb gauge form of a more complicated gauge invariant expression, which is greatly simplified by going to Coulomb gauge, and which represents the energy of a particular type of physical state. The nature of this state is clearest in the Hamiltonian formulation, where the Coulomb gauge Hamiltonian is $H=H_{\text {glue }}+H_{\text {coul }}$, and

$$
\begin{aligned}
& H_{\text {glue }}=\frac{1}{2} \int d^{3} x\left(\mathcal{J}^{-\frac{1}{2}} \boldsymbol{E}^{\mathrm{tr}, a} \mathcal{J} \cdot \boldsymbol{E}^{\mathrm{tr}, a} \mathcal{J}^{-\frac{1}{2}}+\boldsymbol{B}^{a} \cdot \boldsymbol{B}^{a}\right), \\
& H_{\text {coul }}=\frac{1}{2} \int d^{3} x d^{3} y \mathcal{J}^{-\frac{1}{2}} \rho^{a}(x) \mathcal{J} K^{a b}(x, y ; A) \rho^{b}(y) \mathcal{J}^{-\frac{1}{2}},
\end{aligned}
$$

with

$$
\begin{aligned}
K^{a b}(x, y ; A) & =\left[\mathcal{M}^{-1}\left(-\nabla^{2}\right) \mathcal{M}^{-1}\right]_{x y}^{a b}, \\
\rho^{a} & =\rho_{\psi}^{a}+\rho_{\bar{\psi}}^{a}+\rho_{g}^{a}, \\
\mathcal{M} & =-\nabla \cdot \mathcal{D}(A), \quad \mathcal{J}=\operatorname{det}[\mathcal{M}] .
\end{aligned}
$$

Here $\rho_{\psi}^{a}(x)=g \psi_{i}^{\dagger}(x) T_{i j}^{a} \psi_{j}(x), \quad \rho_{\bar{\psi}}^{a}(x)=g \bar{\psi}_{i}(x) T_{i j}^{a} \bar{\psi}_{j}^{\dagger}(x)$ and $\rho_{g}^{a}(x)=-g f^{a b c} A_{k}^{b}(x) E_{k}^{c}(x)$ are the charge density of quarks, antiquarks and gluons, respectively, with $T^{a}, f^{a b c}$ the $\mathrm{SU}(3)$ group generators and structure constants, and $\mathcal{D}_{k}(A)$ is the covariant derivative.

In Coulomb gauge the nonlocal $H_{\text {coul }}$ term derives from solving Gauss's law for the longitudinal color electric field. The position-dependent energy of the physical state created by a set of static quark operators operating, in Coulomb gauge, on the vacuum state can only be due to $H_{\text {coul }}$. For this reason we refer to the energies in (14) as the Coulomb energy of such states. In a continuous time formalism, denote a timelike Wilson line of time extent $t$ by the timeordered exponential

$$
L(\boldsymbol{x}, t)=T \exp \left[i g \int_{0}^{t} d t^{\prime} A_{0}\left(\boldsymbol{x}, t^{\prime}\right)\right],
$$

and the energy is computed from the logarithmic time derivative

$$
\begin{aligned}
\mathcal{E}= & -\lim _{t \rightarrow 0} \frac{d}{d t} \log \left\langle V^{a_{1} a_{2} \ldots a_{N}} V_{b_{1} b_{2} \ldots b_{N}}\right. \\
& \left.\times L_{a_{1}}^{b_{1}}\left(\boldsymbol{x}_{1}, t\right) L_{a_{2}}{ }^{b_{2}}\left(\boldsymbol{x}_{2}, t\right) \ldots L_{a_{N}}^{b_{N}}\left(\boldsymbol{x}_{N}, t\right)\right\rangle .
\end{aligned}
$$

Equation (14) is the Euclidean lattice version of this expression.

We finally note that our construction of physical states with $V$ operators requires that those operators depend on link variables only in a time slice. If we would construct a $V$ operator using, e.g., the gauge transformation to Landau gauge in (17), the resulting operator could not be used to construct a physical state containing a static quark-antiquark pair.

\section{B. The multiplicities of MN and DQP contractions}

Consider initially an isolated set of $N=2^{p} \times 3$ quarks which are in such close proximity that their interactions can be neglected in comparison to their kinetic energies. Even so, either the quark indices are contracted to form a singlet or else we must in addition consider forming a singlet with the help of constituent gluons. For now we consider only the former possibility. In a situation of this sort, we may ask what is the most likely contraction of quark color charges that form the singlet. The simplest possibility is to form $2^{p}$ nucleon states, each a color singlet of three quarks. The number $\Omega_{\mathrm{MN}}$ of ways of making this multi-nucleon grouping is 


$$
\Omega_{\mathrm{MN}}=\frac{N !}{\left(\frac{N}{3}\right) !(3 !)^{N / 3}} .
$$

In this counting, the static quark charges are assumed to occupy different lattice sites and can be treated as distinct objects. The number of ways of grouping a set of $N$ distinct objects into $N / 3$ distinct bins, each containing three objects, is $N ! /(3 !)^{N / 3}$. But since the "bins" are not distinct, and their order is irrelevant, this number must be corrected by dividing by $(N / 3)$ !.

Of course there are a vast number of alternate possibilities. We will not attempt an exhaustive counting, but rather concentrate on the DQP arrangement. This is one particular example of a "one big hadron" state, as it cannot be factorized into subsets of color singlets, and it is easy to count the DQP multiplicity. We first divide $N$ quark charges into $N / 2$ subsets of two quarks each, i.e., diquarks in representation $\overline{\mathbf{3}}$. The multiplicity is

$$
\Omega_{1}=\frac{N !}{\left(\frac{N}{2}\right) ! 2^{N / 2}} .
$$

The next step is to group the $N / 2$ diquarks in $N / 4$ subsets of two diquarks each, with multiplicity

$$
\Omega_{2}=\frac{\left(\frac{N}{2}\right) !}{\left(\frac{N}{4}\right) ! 2^{N / 4}},
$$

proceeding in this way $p$ times until there are only three charged objects left. The total multiplicity is then

$$
\Omega_{\mathrm{DQP}}=\Omega_{1} \Omega_{2} \cdots \Omega_{p}=\frac{N !}{2^{N-3} 3 !} .
$$

Obviously there are far more DQP states than multinucleon states. If the energies of the DQP and multinucleon states are comparable, then purely on the grounds of multiplicity the system is bound to be in a one big hadron state, although not necessarily in a DQP state. But in fact diquark pairings are favored energetically at the perturbative level, and it has been argued that this sort of pairing exists even within nucleons [5]. It could be that DQP states are favored energetically in general among one big hadron states, although this is, of course, far from certain. In any case they constitute a simple alternative to $\mathrm{MN}$ states. Also, the Coulomb energies of both DQP and MN states can be computed numerically and compared at varying densities and spatial arrangements.

\section{SPATIAL ARRANGEMENTS AND COULOMB ENERGIES}

We work in the framework of SU(3) lattice pure gauge theory with the usual Wilson action on a $24^{4}$ lattice volume, at lattice coupling $\beta=5.8$.
Let us consider operators $L_{a}{ }^{b}(i)$ and $M^{a}{ }_{b}(i)$ which transform under the remnant global gauge symmetry as $U_{0}(x)$ and $U_{0}^{\dagger}(x)$ respectively, i.e.,

$$
\begin{aligned}
& L_{a}{ }^{b} \rightarrow L^{\prime}{ }_{a}^{b}=g_{a}{ }^{c} g^{b}{ }_{d} L_{c}{ }^{d} \\
& M^{b}{ }_{a} \rightarrow M^{\prime b}{ }_{a}=g^{b}{ }_{c} g_{a}{ }^{d} M^{c}{ }_{d} \text {. }
\end{aligned}
$$

When contracting two quarks in the $\mathbf{3}$ representation into a diquark in the $\overline{\mathbf{3}}$ representation, and allowing these charges to propagate for one lattice spacing in the time direction, then we are essentially contracting two $U_{0}$ operators into an $M$ operator. Likewise, contracting two diquarks in the $\overline{\mathbf{3}}$ representation into an operator in the $\mathbf{3}$ representation, and propagating for one lattice spacing in the time direction, involves contracting two $M$ operators into an $L$ operator. It will be helpful to introduce the following notation:

(1) Contraction of three $L$ or three $M$ operators to a singlet:

$$
[1: 2: 3] \equiv\left\{\begin{array}{l}
\epsilon^{a_{1} a_{2} a_{3}} \epsilon_{b_{1} b_{2} b_{3}} L_{a_{1}}{ }^{b_{1}}(1) L_{a_{2}} b_{2}(2) L_{a_{3}}^{b_{3}}(3) \\
\epsilon_{a_{1} a_{2} a_{3}} \epsilon^{b_{1} b_{2} b_{3}} M^{a_{1}} b_{1}(1) M^{a_{2}} b_{2}(2) M^{a_{3}} b_{3}(3)
\end{array} .\right.
$$

(2) Contraction of two $L$ operators to an $M$ operator, or contraction of two $M$ operators to an $L$ operator

$$
\{2,3\} \equiv\left\{\begin{array}{l}
\epsilon^{a_{1} a_{2} a_{3}} \epsilon_{b_{1} b_{2} b_{3}} L_{a_{2}}{ }^{b_{2}}(2) L_{a_{3}}^{b_{3}}(3) \\
\epsilon_{a_{1} a_{2} a_{3}} \epsilon^{b_{1} b_{2} b_{3}} M^{a_{2}} b_{2}(2) M^{a_{3}} b_{3}(3)
\end{array} .\right.
$$

As a warm-up exercise, we consider six $(p=1)$ quarks in a plane, arranged in two L-shaped arrangements (indicated by the solid lines) of three quarks, with the groups separated by a lattice distance $R$, as shown in Fig. 1(a). In a $\mathrm{MN}$ arrangement, the three quark indices in each Lshaped subgroup are contracted into a singlet, producing two nucleon states. The energy of this arrangement is obtained, as explained previously, by computing the expectation value of the operator,

$Q_{\mathrm{MN}}(U)=\left[U_{0}(1): U_{0}(2): U_{0}(3)\right] \times\left[U_{0}(4): U_{0}(5): U_{0}(6)\right]$,

with integers 1-6 corresponding to the six quarks shown in Fig. 1(a). The energy per quark is then given by

$$
\mathcal{E}_{q}=-\frac{1}{6} \log \left[\frac{\langle Q(U)\rangle}{Q(\mathbb{1})}\right],
$$

where $Q(U), Q(\mathbb{1})$ correspond to the numerator and denominator of the right-hand side of (14) respectively, with $V$ in the Coulomb gauge being some combination of $\epsilon^{a b c}$ tensors which makes $Q(U)$ a global color singlet. In the DQP arrangement, we pair each quark in one subgroup 


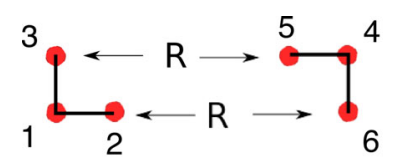

(a) 6 quarks

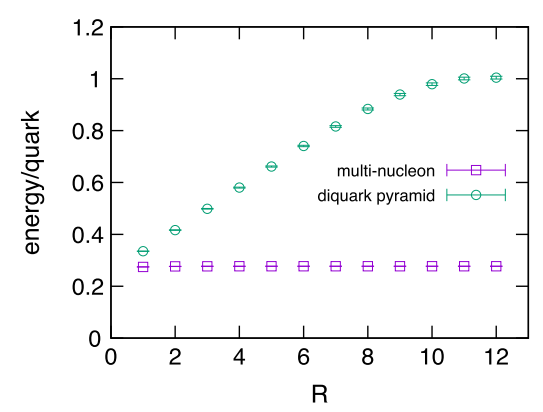

(d) 6 quarks

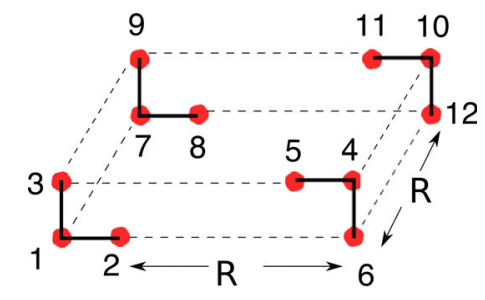

(b) 12 quarks

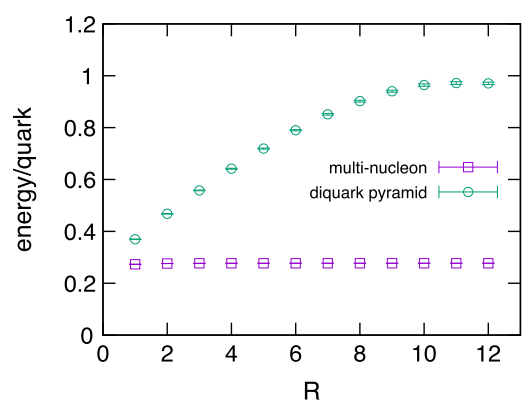

(e) 12 quarks

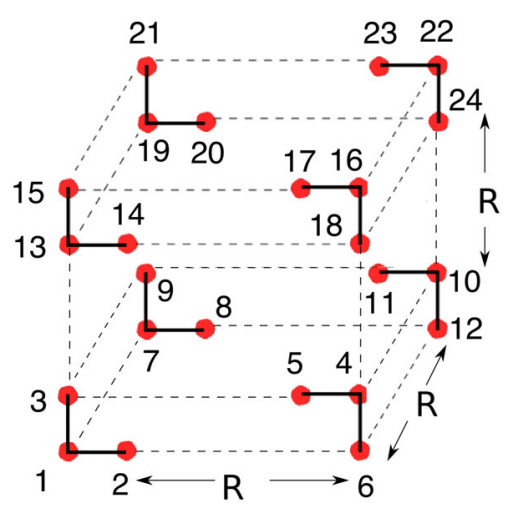

(c) 24 quarks

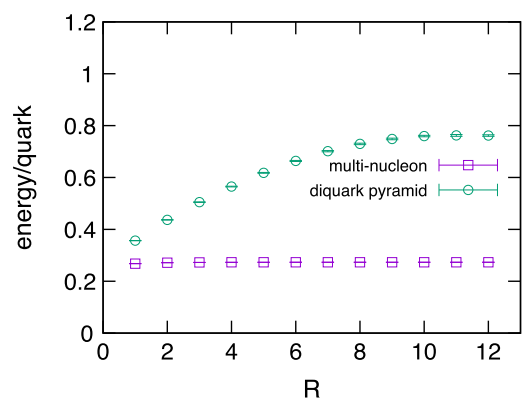

(f) 24 quarks

FIG. 1. Arrangements of (a) 6, (b) 12, and (c) 24 static quarks. In the multinucleon state, triplets of neighboring quarks are contracted into singlets. In the diquark pyramid the colors are contracted as specified in Eqs. (33)-(35), respectively. The Coulomb energies per quark, as a function of the separation $R$, are displayed for the (d) 6, (e) 12, and (f) 24 quark arrangements.

with a quark in the other subgroup to form three diquarks in the $\overline{\mathbf{3}}$ representation, and then contract these three diquarks into a singlet as follows:

$$
\begin{aligned}
Q_{\mathrm{DQP}}(U)= & {\left[\left\{U_{0}(1), U_{0}(4)\right\}:\left\{U_{0}(2), U_{0}(5)\right\}:\right.} \\
& \left.\left\{U_{0}(3), U_{0}(6)\right\}\right] .
\end{aligned}
$$

We compute the Coulomb energies per quark of the $\mathrm{MN}$ and DQP states from (32), as described previously, and find the results shown in Fig. 1(d). These are as expected. In the MN case, the energies are almost independent of the separation $R$ between the L-shaped nucleons. In the DQP case, the energy rises linearly with separation (until the data begin to flatten out due to the finite lattice volume) and is strongly disfavored energetically compared to the MN configuration.

The next possibilities are $12(p=2)$ and $24 p=3$ quarks, with L-shaped groups of three quarks placed in the arrangements shown in Figs. 1(b) and 1(c) respectively. Again the $Q_{\mathrm{MN}}$ operator is constructed by contracting indices in each L-shaped group to form a singlet and then taking the product. The $Q_{\mathrm{DQP}}$ operator is created by first contracting pairs of quark charges to form diquarks in the $\overline{\mathbf{3}}$ representation, then contracting pairs of $\overline{\mathbf{3}}$ diquarks into $\mathbf{3}$ combinations, and so on until finally contracting the three remaining operators into singlets. Shortening the notation further to let an integer $i$ denote the link variable $U_{0}(i)$ associated with the $i$ th static quark, the choice of contractions is, for the 12-quark combination

$$
\begin{aligned}
Q_{\mathrm{DQP}}(U)= & {[\{\{1,4\},\{7,10\}\}:\{\{2,5\},\{8,11\}\}} \\
& :\{\{3,6\},\{9,12\}\}],
\end{aligned}
$$

and for the 24-quark combination

$$
\begin{aligned}
Q_{\mathrm{DQP}}(U)= & {[\{\{\{1,4\},\{7,10\}\},\{\{13,16\},\{19,22\}\}\}} \\
& :\{\{\{2,5\},\{8,11\}\},\{\{14,17\},\{20,23\}\}\}: \\
& \{\{\{3,6\},\{9,12\}\},\{\{15,18\},\{21,24\}\}\}] .
\end{aligned}
$$

We compute energies $\mathcal{E}_{q}$ per quark from $-\log [\langle Q(U)\rangle /$ $Q(\mathbb{1}]$, and dividing by the number of quarks, either 12 or 24 in these cases. The energies of the corresponding DQP and MN states for these 12 and 24 quark states vs $R$ are shown in Figs. 1(e) $(p=2)$ and 1(f) $(p=3)$ respectively. Again this is what one would expect; in both cases the MN state is 


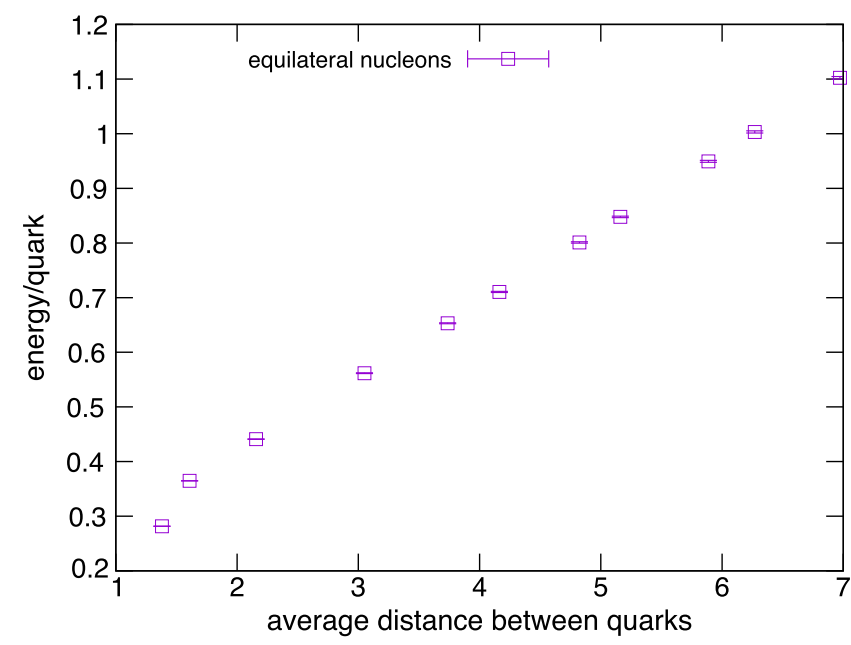

FIG. 2. The Coulomb energies of a nucleon state consisting of three static quarks in an arrangement as close as possible (within the constraints of the lattice structure) to an equilateral triangle. The figure displays the energies of such states as a function of the average interquark separation in the state.

independent of the separation $R$, while the energy of the DQP state rises linearly with $R$.

Rather than keep three quarks in a nearest-neighbor L-shaped configuration, we may also compute the Coulomb energy of a single three quark nucleon as a function of average quark separation within the nucleon. In this calculation we arrange the quarks to lie as closely as possible (within the constraints of the lattice structure) on an equilateral triangle, with the average quark separation defined as being $R=\left(d_{12}+d_{23}+d_{31}\right) / 3$, where $d_{i j}$ is the (straight-line) distance between quarks $i, j$ in the nucleon. Once again, and unsurprisingly, the Coulomb energy per quark rises linearly with $R$, as seen in Fig. 2.

\section{COULOMB ENERGY IN A DENSE MEDIUM}

Instead of keeping the number of quarks fixed and varying the separation between nucleons, as in the previous section, we next keep the density fixed at one quark per lattice site in a rectangular volume, and vary the size of the rectangular volume containing the quarks. In this setup we choose to average over all possible color contractions of the $\mathrm{MN}$ and DQP type. In other words, in the MN case, we group the quarks into $2^{p}$ randomly chosen sets of three, contracting the indices in each group into a singlet. In the DQP scheme we group the quarks into $2^{p-1} \times 3$ randomly chosen sets of two quarks, contracting the indices to form a $\overline{\mathbf{3}}$ combination. These diquarks are then randomly grouped into $2^{p-2} \times 3$ sets of two diquarks, contracting the indices in each set into a 3 combination, and so on until only three $\overline{\mathbf{3}}$ or 3 combinations are available, and these are finally grouped into a singlet. We then compute the Coulomb energy from correlation functions of timelike link variables, as explained above, and divide by the number of quarks to obtain the energy per quark. In this calculation the quarks are arranged in a rectangular volume of $i \times j \times k=2^{p} \times 3$ lattice units, with each lattice site in the volume occupied by one quark. At each data-taking sweep through the lattice we compute the observable in each $i j k$ rectangular subvolume of the lattice, choosing a different random grouping in each subvolume.

There is, of course, a maximum density of quarks that can be placed on the lattice, e.g., in the staggered lattice formulation with one flavor, the maximum is three quarks per site. This maximum density is a lattice artifact associated with quarks occupying the same site. Taking the density to be one quark/lattice site is a compromise between having the highest density possible, and avoiding the onset of this kind of lattice artifact, which would also invalidate the counting shown in Eqs. (24)-(27).

As an example, the expectation value of the $Q_{\mathrm{DQP}}(U)$ operator, for the $3 \times 2 \times 4$ arrangement corresponding to Fig. 1(c) with $R=1$, involves taking the expectation value of the operator shown in (35), but averaged over all permutations of the quark numbers shown. Likewise, for the $3 \times 2 \times 1$ arrangement corresponding to Fig. 1(a) at $R=1$, the energy of the multinucleon case is extracted from the expectation value of the operator $Q_{\mathrm{MN}}(U)$ as shown in (31), but averaged over all permutations of the quarks in this expression.

The results of this calculation, shown in Fig. 3 for each $i j k$ arrangement, are a little surprising. Consider the MN contractions. If the quarks are divided at random in groups of 3, then the average separation between quarks in each group, denoted by $R$, ought to grow with the volume. Yet the dependence of energy per quark (= energy density $\mathcal{E}_{q}$ ) turns out to be only mildly dependent on the $i \times j \times k$ volume, even as we go from 6 quarks at $i j k=(6,1,1)$ and $R=2.33$, where we find $\mathcal{E}_{q}=0.41$, to 768 quarks at $i j k=$ $(12,8,8)$ and $R=6.23$ with $\mathcal{E}_{q}=0.44$. In Fig. 4 we plot $\mathcal{E}_{q}$ vs $R$ for the MN states of quarks in the rectangular volume, as compared to the energy per quark vs $R$ for three isolated quarks in a roughly equilateral arrangement, which was already displayed in Fig. 2. We have also computed the energy of single nucleon states extracted from the $i j k$ volumes, denoted "nucleons in vacuum" in the figure. Here we have computed the single nucleon energies by contractions $[a: b: c]$ for quarks $a, b, c$ chosen at random in the $i j k$ volumes, and averaged over the possible choices of $a$, $b, c$. As opposed to the MN states we do not take the product of such contractions, but only take the logarithm of the expectation value of single nucleon contractions to obtain a single nucleon energy, which we have again plotted against the average quark separation. These energies represent the energies of nucleons in a vacuum, rather than in a medium, although not necessarily in an equilateral arrangement. It can be seen that the energies of the "nucleons in vacuum" are roughly parallel, as a function of average quark separation, to the energies of quark triplets in an equilateral configuration. 


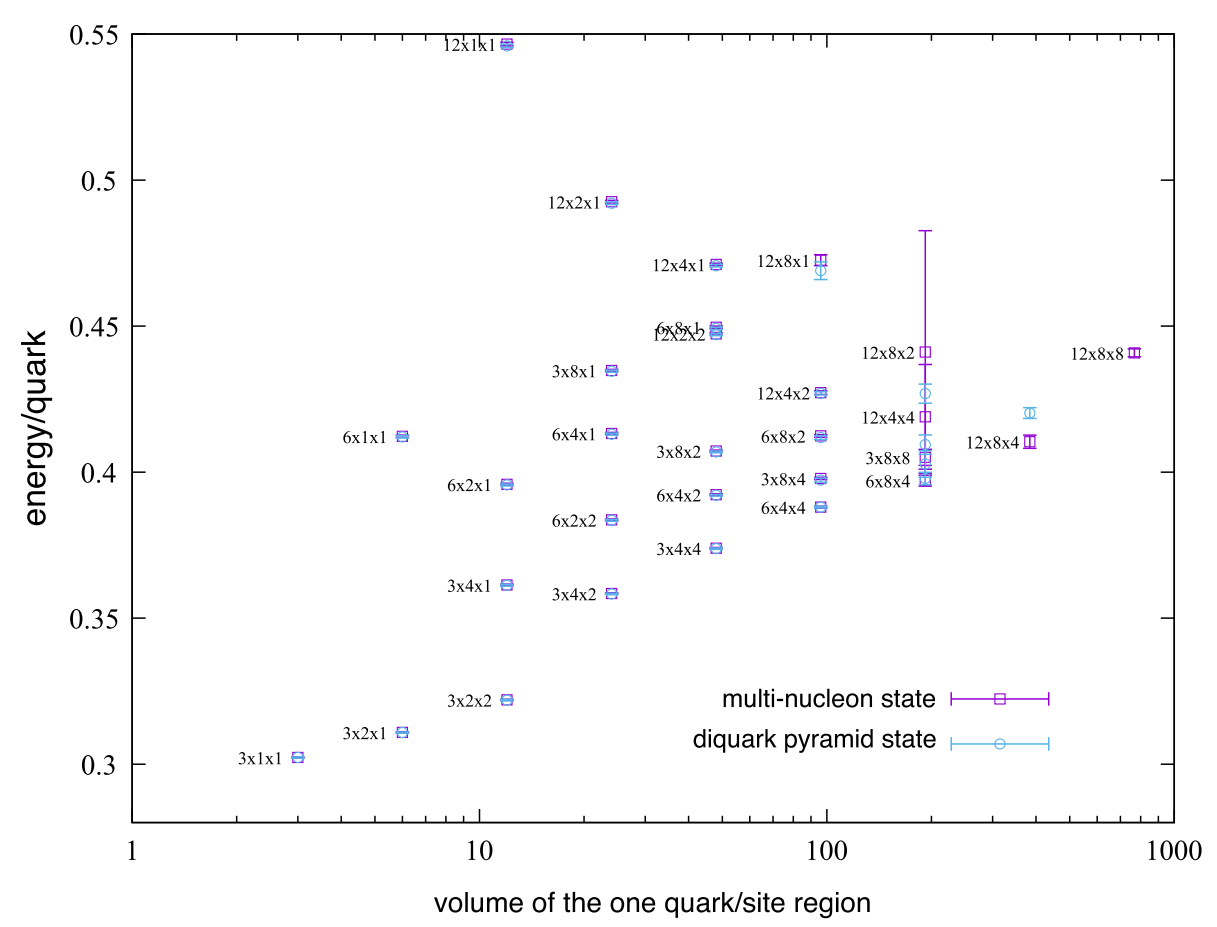

FIG. 3. Coulomb energies per quark for dense quark systems (one quark/site) in an $i \times j \times k$ volume, plotted versus the volume. The multinucleon state groups the quarks at random into sets of 3, contracting each triplet into a singlet. The diquark pyramid contractions are also randomized, as described in the text.

What is clear from Fig. 4 is that the average Coulomb energy per quark in a nucleon embedded in a dense medium is only mildly dependent on the average separation of the quarks within a nucleon, whereas the energy clearly increases linearly with separation for an isolated triplet

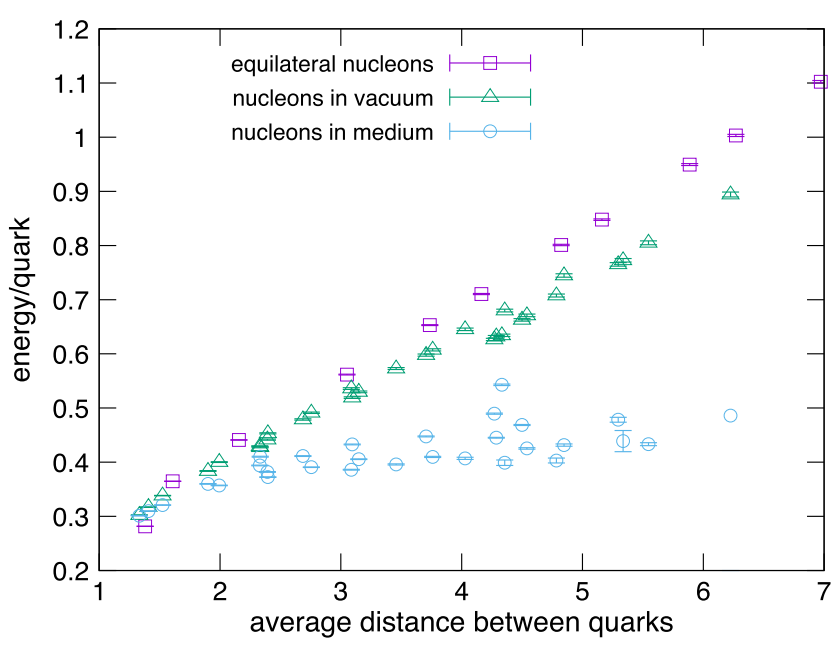

FIG. 4. The energy/quark in the multinucleon states (open circles) shown in the previous figure, plotted against the average separation between quarks in those nucleons (random triplets chosen at random in the $i j k$ volume, contracted to a singlet). Also plotted are the energy per quark of isolated nucleons in vacuum (open squares) in a (nearly equilateral) arrangement, which was already plotted in Fig. 2, and of individual random nucleons (open triangles), selected as described in the text. of quarks. Since this is the central result of our work, it may be worth repeating: in the MN case we choose sets of three quarks in the $(i j k)$ volume at random, and of course this means that the average interquark separation of quarks within each nucleon increases as the rectangular volume increases. The surprise is that this increase in separation in only very weakly reflected in the Coulomb energy per quark. If the quarks were dynamical this would not necessarily be a surprise; the phenomenon might be expected from a Debye screening process of some kind. But with static quarks, and an energy which derives from the instantaneous and nonlocal Coulomb term in the Coulomb gauge Hamiltonian, it is hard to see how there can be any Debye-like screening process. So this result we consider a surprise. We do not, at present, have an explanation for this effect.

The energy/quark $\mathcal{E}_{q}$ for the random DQP arrangements described above is also displayed, together with the previous $\mathrm{MN}$ results, in Fig. 3. What is again a little surprising, at least to us, is that the $\mathrm{MN}$ and $\mathrm{DQP}$ energies per quark are quite comparable. The DQP arrangement of dense static quarks should be preferred, thermodynamically, just on considerations of multiplicity, as noted in Sec. II.

Finally we have studied how $\mathcal{E}_{q}$ changes (in lattice units at $\beta=5.8$ ) as the density of quarks in the $i j k$ volumes are varied away from the density of one static quark per lattice site. For this purpose we start with 48 quarks in an $(i j k)=$ $(3,4,4)$ arrangement. Keeping the number of quarks fixed 
at 48 , we increase the volume by adding, at each step, one unit to the length of the volume in the $x, y, z$ directions, in that order. In other words, we increase the volume in the following order:

$$
\begin{aligned}
& (i+1, j, k),(i+1, j+1, k),(i+1, j+1, k+1), \\
& \quad(i+2, j+1, k+1), \\
& (i+2, j+2, k+1),(i+2, j+2, k+2), \\
& \quad(i+3, j+2, k+2), \ldots
\end{aligned}
$$

The 48 quarks are placed at random in each of the larger $\left(i^{\prime}, j^{\prime}, k^{\prime}\right)$ volumes, with the MN and DQP contractions constructed as before. We observe in Fig. 5 that the energy per quark does increase somewhat as the density decreases (volume/quark increases), as one might expect, but the increase in energy in the DQP and (especially) the MN cases is comparatively modest, given an order-of-magnitude reduction in the quark density.

In the Introduction we mentioned the sign problem in connection with high baryon density, which makes a conventional Monte Carlo calculation (e.g., via reweighting) unfeasible due to sign cancellations. Inspection of Fig. 3, which displays on the $y$-axis the logarithm of $\langle Q(U) / Q(\mathbb{1})\rangle$ divided by the number of quarks, makes it clear that $\langle Q(U) / Q(\mathbb{1})\rangle$ itself must have extraordinarily small values for our larger volumes of quarks, and the question is how it is possible that such minute values are not swamped by statistical error. The answer is that these very small values are not, for the most part, due to delicate sign cancellations among measured values of $\mathrm{O}(1)$, but rather come mainly from the very small magnitude of each measured value. This is illustrated for the $3 \times 4 \times 4$ volume by the histogram of $Q(U) / Q(\mathbb{1})$ values shown in Fig. 6. The set consists of 100000 values obtained at $\beta=5.8$ on a

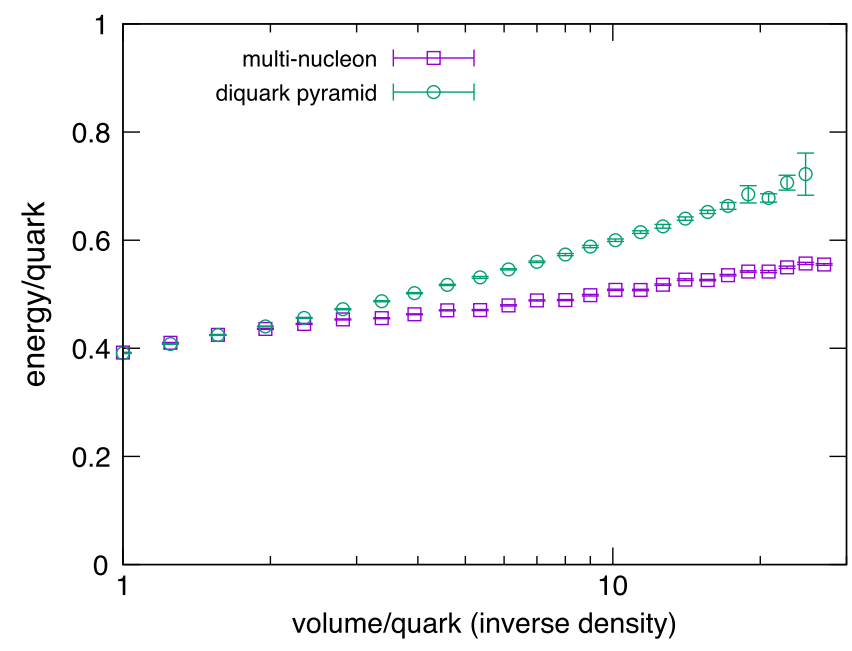

FIG. 5. The energy per quark of a system of 48 quarks in which the density of the system is varied by increasing the volume. The plot is energy per quark vs inverse density.

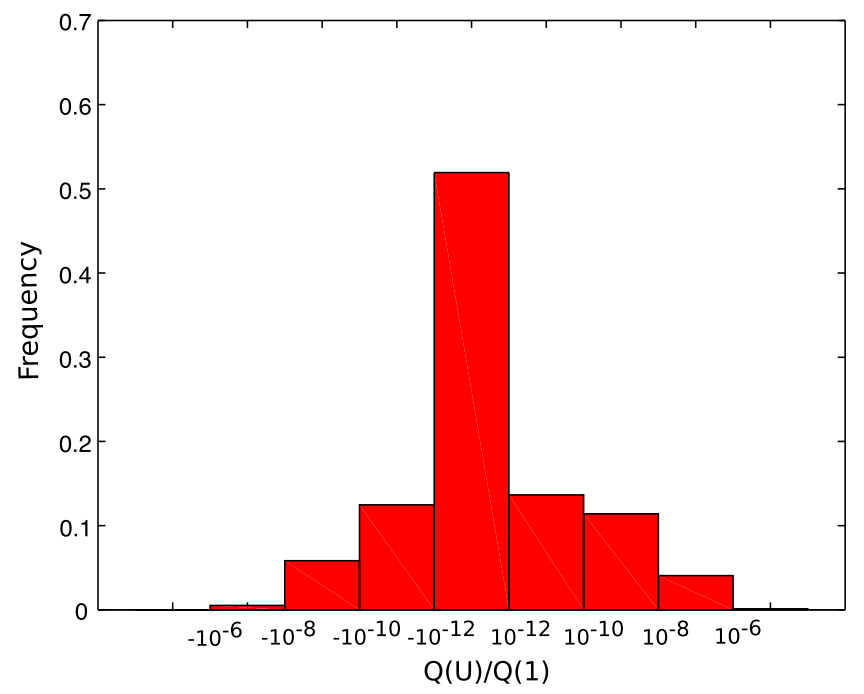

FIG. 6. Histogram of 100000 values of $Q(U) / Q(\mathbb{1})$ obtained for 48 static quarks in a $3 \times 4 \times 4$ volume. The central bin shows the frequency of measured values lying between $-10^{-12}$ and $+10^{-12}$, the adjacent bin to the right is the frequency of measured values in the range $10^{-12}$ to $10^{-10}$, and so on. Note the small magnitude of these values and the asymmetry in the frequencies of positive and negative values.

$10^{4}$ lattice. The average value in this case is $7.06 \times 10^{-9}$, and the asymmetry between positive and negative values, which results in this nonzero average, is obvious at a glance.

\section{CONCLUSIONS}

Studies of the Coulomb energy in static quark systems have generated some unexpected results. In the past it was found that the Coulomb energy in a static quark-antiquark pair rises linearly with quark separation [3,4]; contains a Lüscher term [4]; and (here is the surprise) is arranged into a flux tube which is somewhat more narrow than the minimal energy configuration [6], rather than being spread out over all space as one might naively expect.

We have now investigated the Coulomb energy of a system of $2^{p} \times 3$ static quarks, at a density of one quark per lattice site in a rectangular volume, with colors contracted into either a set of $2^{p}$ "nucleons," the MN state, or into a diquark "pyramid," the DQP state, which cannot be factorized into subsets of color singlets. We average over the possible selections of three quarks into nucleon subsets, or the choice of quark pairs contracted into diquarks in the DQP state, as explained previously. What we have found is that the energy per quark, in either the MN or DQP states, is only very weakly dependent on the rectangular volume containing the quarks. The reason this was unexpected, especially in the MN case, is that while the average separation between quarks in a nucleon rises with volume, this is not reflected in a corresponding linear rise in the energy per quark. This behavior of the energy of a nucleon 
in a dense medium contrasts sharply with the corresponding energetics of an isolated nucleon with static quarks, where the energy per quark does rise linearly with quark separation.

In a plasma of dynamical quarks, this insensitivity of energy/quark to the volume of the ensemble of quarks would not be especially surprising and would be explained via some Debye-like screening mechanism in the quark plasma. But since we are dealing with an ensemble of static quarks, whose Coulomb interactions presumably arise from the instantaneous nonlocal term in the Coulomb gauge Hamiltonian $H_{\text {coul }}$ of Eq. (20), it is difficult to appeal to such screening mechanisms. We appear to be finding some unexpected property of the Coulomb energy of a dense ensemble of quark charges, a kind of "screening without screening," such that the long-range interactions of static quarks are somehow damped without corresponding quark motion. Exactly how this effect emerges from the nonlocal kernel $K^{a b}(\boldsymbol{x}, \boldsymbol{y} ; A)$ of $(21)$ is unclear at the moment. The data, however, indicate that the Coulomb energy density of a state at fixed baryon number density is fairly insensitive to the total number of quarks, regardless of how the color indices are contracted. This is obviously a nonperturbative effect.
It should be understood that although the states we are constructing are physical states, whose interaction energy is the Coulomb energy, these are not necessarily the minimal energy states of a dense quark system. For example, the analogous states of a quark-antiquark pair have a string tension, due to the Coulomb interaction, which is about four times the asymptotic string tension. Nevertheless, qualitative features of such states, such as the linear potential, the existence of a flux tube, and the Lüscher term, persist in the minimal energy state. It is possible that screening without screening is a feature of the minimal energy version of a heavy dense quark ensemble, which may have implications also for light quarks at finite densities. We leave this possibility for future study.

It should also be noted that in this study we have worked throughout with a relatively coarse lattice at $\beta=5.8$. It would be of interest to hold the density fixed in physical units on finer lattices, and also to study further the variation in energy density with quark density. We hope to obtain results along these lines in a later investigation.

\section{ACKNOWLEDGMENTS}

This work is supported by the U.S. Department of Energy under Grant No. DE-SC0013682.
[1] G. Aarts, F. Attanasio, B. Jager, and D. Sexty, J. High Energy Phys. 09 (2016) 087.

[2] J. Langelage, M. Neuman, and O. Philipsen, J. High Energy Phys. 09 (2014) 131.

[3] J. Greensite and S. Olejnik, Phys. Rev. D 67, 094503 (2003); Y. Nakagawa, A. Nakamura, T. Saito, H. Toki, and D. Zwanziger, Phys. Rev. D 73, 094504 (2006).
[4] J. Greensite and A. P. Szczepaniak, Phys. Rev. D 91, 034503 (2015)

[5] M. Anselmino, E. Predazzi, S. Ekelin, S. Fredriksson, and D. B. Lichtenberg, Rev. Mod. Phys. 65, 1199 (1993); D. B. Lichtenberg, W. Namgung, E. Predazzi, and J. G. Wills, Phys. Rev. Lett. 48, 1653 (1982).

[6] K. Chung and J. Greensite, Phys. Rev. D 96, 034512 (2017). 